\title{
PRESENCE OF ALBUMIN IN ERYTHROCYTE LYSATE OF AUTOIMMUNE HAEMOLYTIC ANAEMIA PATIENTS OBSERVED IN ELECTROPHORETOGRAM
}

\author{
Mathew John ${ }^{1 *}$, Evelyn Maria ${ }^{2}$, Minu Merin Sabu ${ }^{3}$, Karthika Asok ${ }^{3}$, Sinju R ${ }^{4}$ \\ ${ }^{1}$ Scientist, Jubilee Centre for Medical Research, Jubilee Mission Medical College \& Research Institute, Thrissur \\ 680005 \\ ${ }^{2}$ Research Assistant, Jubilee Centre for Medical Research, Jubilee Mission Medical College \& Research \\ Institute, Thrissur 680005 \\ ${ }^{3}$ Project fellow, Jubilee Centre for Medical Research, Jubilee Mission Medical College \& Research Institute, \\ Thrissur 680005 \\ ${ }^{4}$ Tutor, Department of Biochemistry, Jubilee Mission Medical College \& Research Institute, Thrissur 680005
}

Article Info: Received 28 April 2019; Accepted 28 May. 2019

DOI: https://doi.org/10.32553/jbpr.v8i3.603

Address for Correspondence: Mathew John, Scientist, Jubilee Centre for Medical Research, Jubilee Mission Medical College \& Research Institute, Thrissur 680005

Conflict of interest statement: No conflict of interest

\section{ABSTRACT:}

Autoimmune haemolytic anaemia is a condition in which autoantibodies present against self erythrocytes which leads to severe haemolysis and loss of haemoglobin. In the present study we compared the electrophoretic band patterns between control and autoimmune haemolytic anaemia and non autoimmune iron deficiency anaemia erythrocyte lysate samples. We observed the presence of a band which was specific to autoimmune haemolytic erythrocyte lysates in the region of $70 \mathrm{Kd}$ which was later identified as albumin and was distinctly absent in control as well as iron deficiency non autoimmune haemolytic samples. In addition, we have noticed that the catalase in the region of $60 \mathrm{Kd}$ was found to be decreased in its band intensity in haemolytic anaemia samples compared to control. The presence of both albumin and catalase in the respective positions in the electrophoretic gel was confirmed by in gel trypsin digestion followed by mass spectrometry. The other erythrocyte proteins visible in the region of $25 \mathrm{Kd}$ were found to have an overall decrease in band intensity in haemolytic anaemia samples compared to control. The specific presence of albumin in autoimmune haemolytic erythrocyte lysate is the main highlight of the work which was not reported till date and further studies could reveal the exact role of albumin in autoimmune haemolytic conditions.

Key words: Autoimmune haemolytic anaemia, erythrocytes, electrophoresis, albumin, catalase

\section{INTRODUCTION}

Autoimmune haemolytic anaemia (AIHA) occurs when the immune system has antibodies that attack the erythrocytes which causes a decline in the number of red blood cells, leading to haemolytic anaemia. AlHA is characterised by haemolysis mediated by autoantibodies directed against self erythrocytes with the incidence of 1-3 per 100,000 per year with $11 \%$ mortality $(1,2)$. AlHA are characterised as warm and cold reactive or of mixed type based on sensitivity to warm or cold reactive Immunoglobulin G (IgG) (3-5). The laboratory diagnosis of AlHA depends on result of direct antiglobulin test which shows positivity with anti IgG, which is referred as Direct Coombs test (DCT). These antibodies are usually IgG which are capable of fixing complement, and are detected by Coombs test. The direct antiglobulin Coombs test is based on specific antibody to IgG and C3d, the third component of complement. If $\mathrm{C} 3 \mathrm{~d}$ or IgG are present on the erythrocyte membrane, the result is a visible agglutination by cross linking erythrocytes. It can be idiopathic or secondary to 
lymphoprolifeartive syndromes, autoimmune diseases, infections and tumors (6). AlHA may develop gradually with concomitant physiological compensation or may have a fulminant onset with profound life threatening anaemia. AlHA is an uncommon entity that presents diagnostic, prognostic and therapeutic dilemmas which is attributed to the significant variations in rate of hemolysis and associated diseases which may add to the clinical heterogeneity (7). It is important to consider that degree of anaemia depends on severity of erythroblastic response. In fact, patients with reticulocytopenia, reported to occur in some $20 \%$ of adults and $39 \%$ of children $(8,9)$. Erythrocyte destruction may occur by a direct lysis through the sequential activation of the final components of the complement cascade which are mediated by membrane attack complex or antibody dependent cell mediated toxicity (10).Approximately 7 to $8 \%$ of AlHA have serological findings characteristics of warm AIHA. Most of AlHA reported in adults are of warm type and very few cases reported as cold type. The warm and cold type are classified based on the thermal optimum used to detect anti erythrocyte antibody (11).

In the present study we aimed to study the difference in erythrocyte proteins between control and haemolytic anaemia by SDS-PAGE. It is worthwhile to consider that erythrocytes could be influenced by plasma proteins such as albumin along with globulin. Albumin and globulin are the predominant plasma proteins which are capable of maintaining the morphological as well as physiological characteristics of erythrocytes. Albumin maintains the biconcave shape of the normal erythrocyte and decreases both osmotic and mechanical fragilities of erythrocytes. The observed protection of albumin against cell lysis may be related to the ability of the protein to stabilize the biconcave shape. If albumin affects cell geometry, it may alter cell volume and also its density (12). It was reported in a study that albumin had a synergestic effect with $y$ - globulin in decreasing erythrocyte sedimentation rate (13, 14).

\section{Materials and Methods}

All the chemicals used for the experiments were purchased from HiMedia. Protease inhibitor cocktail was purchased from Sigma. For electrophoresis experiments the reagents and electrophoretic apparatus were obtained from BioRad.

The blood samples were collected from the central lab of the host institute and the study is approved by Institutional ethics committee. The study groups were divided into 3 and mentioned as follows.

Group 1: Normal healthy control

Group 2: Coombs test positive warm type autoimmune haemolytic anemia

Group 3: Coombs test negative iron deficiency anaemia and non autoimmune

The healthy control was selected after confirmation of normal values of haematological and other routine blood parameters. The control samples had haemoglobin $(\mathrm{Hb})$ values 13.8 and 14.5 and the non autoimmune iron deficiency samples had 7.5, 9.6 and $8.8 \mathrm{~g} / \mathrm{dL}(\mathrm{Hb})$. The autoimmune samples positive for Coombs test had haemoglobin values 4.5 and $3.8 \mathrm{~g} / \mathrm{dL}$. All the selected samples were of adult type with age between 40 and 65 years including only males. The females were excluded from the study as they have lowered normal reference range for erythrocytes and haemoglobin due to physiological reasons.

\section{Erythrocyte lysate preparation}

Erythrocyte lysate preparation was done according to Arias et.al (15) with appropriate modifications. Approximately $1 \mathrm{~mL}$ of whole blood was diluted with $4 \mathrm{~mL}$ of cocktail $-1(120 \mathrm{mM} \mathrm{NaCl}, 10 \mathrm{mM}$ EDTA, $5 \mathrm{mM}$ sodium citrate and $5 \mathrm{mM}$ Tris- $\mathrm{HCl}, \mathrm{pH}$ 7.4. The mixture was centrifuged at $3000 \mathrm{rpm}$ for 10 minutes. The supernatant was discarded and the pellet was washed 3 times in $150 \mathrm{mM} \mathrm{NaCl}$, $5 \mathrm{mM}$ Tris $\mathrm{HCl}, \mathrm{pH}$ 7.4. The residual pellet was made up to $2 \mathrm{~mL}$ in cocktail 2 consisting of $120 \mathrm{mM} \mathrm{NaCl}$ and $5 \mathrm{mM}$ Tris $\mathrm{HCl}, \mathrm{pH} 7.4$ followed by addition of 2 $\mathrm{mL}$ distilled water. The samples were incubated at $37^{\circ} \mathrm{C}$ for 10 minutes followed by centrifugation at $13,000 \mathrm{~g}$ for 60 minutes at $4^{\circ} \mathrm{C}$. The resulting supernatant was added in re suspension solution at $1: 1 \mathrm{v} / \mathrm{v}$. The re suspension solution consisted of $300 \mathrm{mM} \mathrm{NaCl}, 100 \mathrm{mM}$ Tris $\mathrm{HCl}, 0.5 \%$ SDS, $1 X$ protease inhibitor cocktail and $0.5 \mathrm{mM}$ DTT. This was followed by another centrifugation at $13,000 \mathrm{~g}$ for 10 minutes to remove any other debris. The clear supernatant erythrocyte lysate was 
resuspended in $100 \mathrm{mM}$ Tris $\mathrm{HCl}, \mathrm{pH} 7.4$ at 1:1 v/v. The erythrocyte lysate of respective groups was further subjected to protein quantitation using BCA method. After normalising the protein concentration to $100 \mu \mathrm{g}$ in $25 \mu \mathrm{L}$, the samples were loaded to respective wells in a $4-10 \%$ gradient SDS-PAGE followed by Coomassie staining and bands were visualised in white field in a transilluminator and image was photographed.

\section{Mass spectrometry}

The two bands in the region between $75 \mathrm{Kd}$ and 50 $\mathrm{Kd}$ was excised from the gel and subjected to in gel trypsin digestion followed by MALDI-TOF analysis and peptide sequencing was done and proteins which matched the peptide sequence was analysed using MASCOT score histogram.

\section{Froth height test to assess the catalase activity in erythrocytes}

Erythrocyte lysate supernatant was added to 80 $m \mathrm{~m} \mathrm{H} \mathrm{H}_{2}$. When the substrate $\mathrm{H}_{2} \mathrm{O}_{2}$ was added to erythrocyte lysate, due to catalase activity, $\mathrm{H}_{2} \mathrm{O}_{2}$ was converted to $\mathrm{O}_{2}$ which was generated as froth. The height in froth formation was indicative of the catalase activity in erythrocytes.

\section{Results}

Comparative SDS-PAGE of control and haemolytic anaemia erythrocyte lysate samples

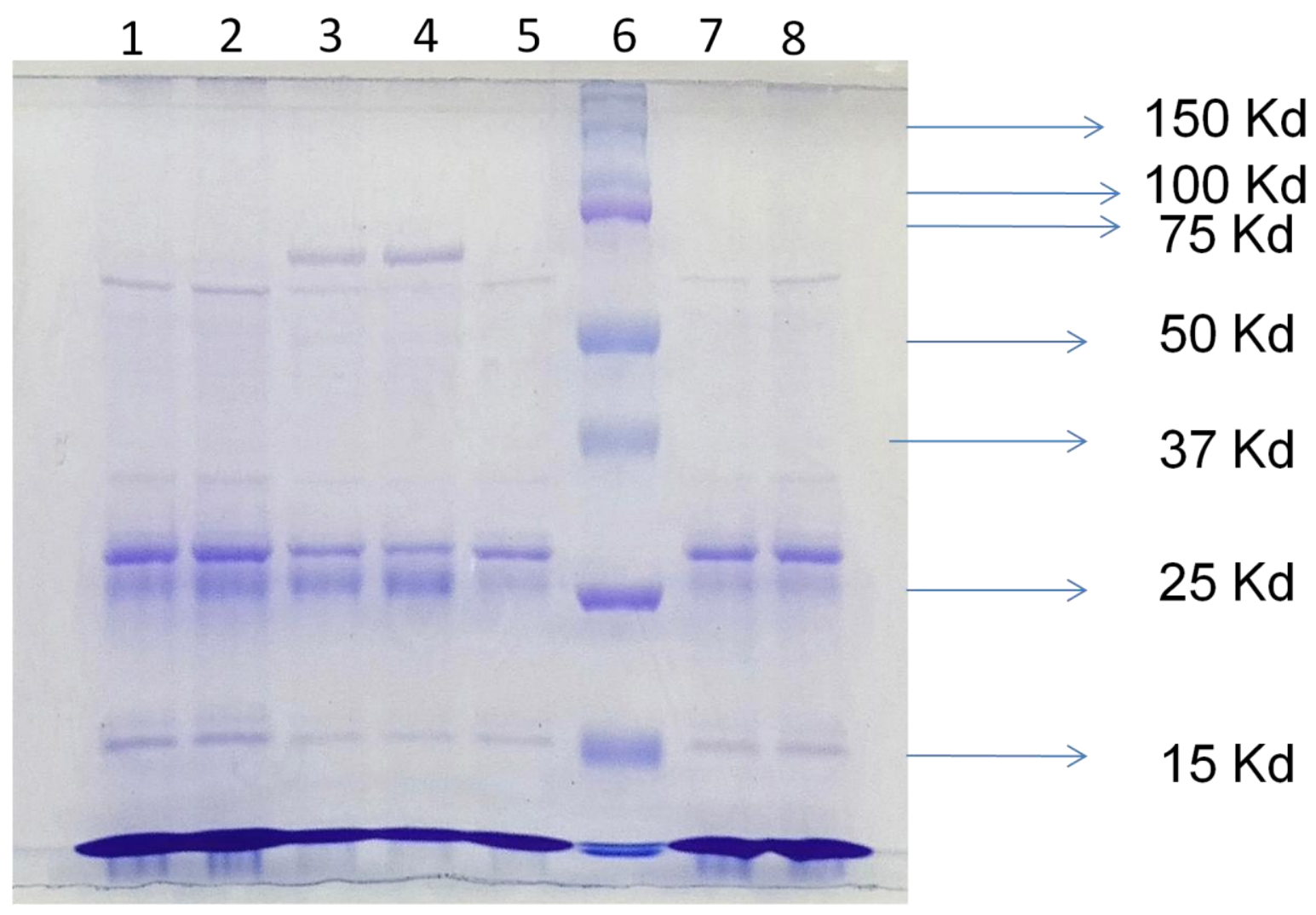

Figure 1:

Figure - 1 depicted the SDS-PAGE of erythrocyte lysate of control and haemolytic anaemia patients. The electrophoretogram of erythrocyte lysate of control, Coombs test positive autoimmune haemolytic anaemia and Coombs test negative non autoimmune iron deficiency anaemia revealed that there were noticeable differences between control and haemolytic anaemia erythrocyte proteins. From the pre stained protein marker position between $75 \mathrm{Kd}$ and $50 \mathrm{Kd}$, there appeared one prominent band in AlHA samples in lane 3 and 4 which correspond to AlHA and was absent in control and non autoimmune iron deficiency anaemia samples in lane 1 and 2 and lane 5, 7 and 8. It was also found another band between $75 \mathrm{Kd}$ and $50 \mathrm{Kd}$ which was more prominent in control samples compared to AlHA and non autoimmune haemolytic anaemia (NAIHA). Both these bands correspond to albumin and catalase respectively and identified by mass 
spectrometry experiment, MALDI-TOF followed by MASCOT software for peptide mapping and sequencing. The other bands visible in the region near $25 \mathrm{Kd}$ could be stomatin, aquaporin and peroxiredoxin and noticed that band intensity in these regions correspond to the above erythrocyte proteins were higher in control samples compared to haemolytic anaemia. The band in the region of $15 \mathrm{Kd}$ could be beta globin chain of haemoglobin and observed that band intensity was higher in control samples compared to haemolytic samples.

Mass spectrometry data of proteins identified by MALDI-TOF

MALDI-TOF details of Albumin

Table 1:

\begin{tabular}{|l|l|l|l|}
\hline Protein hits code & Peptide sequence & $\begin{array}{l}\text { Proteins matching with peptide } \\
\text { sequence }\end{array}$ & Mass \\
\hline AoAoC4DGB6 & K.VFDEFKPLVEEPQNLIK.Q & Albumin & 71177 \\
\hline ALBU & K.VFDEFKPLVEEPQNLIK.Q & Albumin & 71317 \\
\hline BRDPP6 & K.VFDEFKPLVEEPQNLIK.Q & Similar in sequence to albumin & 72370 \\
\hline F6KPG5 & K.VFDEFKPLVEEPQNLIK.Q & Albumin fragment & 68484 \\
\hline A0A087WWT3 & K.VFDEFKPLVEEPQNLIK.Q & Albumin & 46430 \\
\hline C9JKR2 & K.VFDEFKPLVEEPQNLIK.Q & Albumin isoform & 49568 \\
\hline
\end{tabular}

Table - 1 described the MALDI-TOF details of albumin. The MASCOT search histogram revealed that the peptide sequence identified after in gel tryptic digestion matched with that of albumin.

MALDI-TOF details of Catalase

Table 2:

\begin{tabular}{|l|l|l|l|}
\hline Protein hits code & Peptide sequence & Proteins matching with peptide sequence & Mass \\
\hline CATA human & R.AFYVNVLNEEQR.K & Catalase & 59947 \\
\hline 4DWK8 & R.AFYVNVLNEEQR.K & Catalase & 53482 \\
\hline Q8TAK2 & R.AFYVNVLNEEQR.K & Catalase fragment & 26093 \\
\hline
\end{tabular}

Table - 1 described the MALDI-TOF details of catalase. The MASCOT search histogram revealed that the peptide sequence identified after in gel tryptic digestion matched with that of catalase.

Froth height test to assess the catalase activity in erythrocyte lysate between control and haemolytic anaemia samples

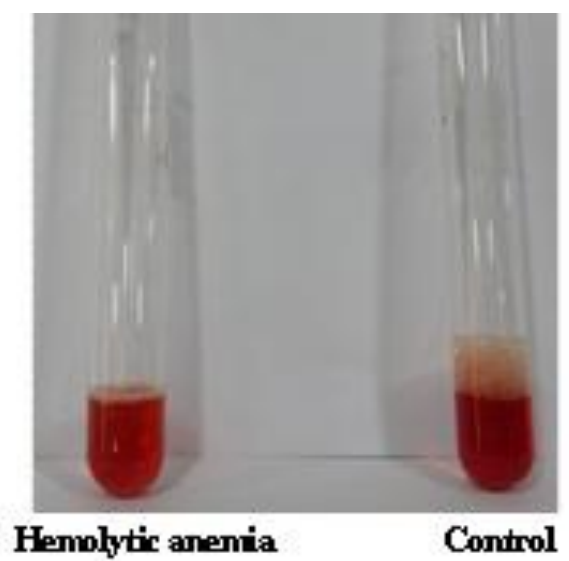

Figure 2: 
Froth height in control erythrocyte lysate $-0.5 \mathrm{~cm}$

Froth height in autoimmune haemolytic erythrocyte lysate $-0.1 \mathrm{~cm}$

Figure- 2 was a simple test to assess the catalase activity of erythrocytes of control and autoimmune haemolytic anaemia. The result showed that when $\mathrm{H}_{2} \mathrm{O}_{2}$ was added to erythrocyte lysate there was generation of froth due to the formation of oxygen. The froth formation was measured in scale and height in froth formation was indicated. The froth formation was indicative of the catalase activity of erythrocytes. From the figure it was visualised that there was distinctive decrease in froth formation in autoimmune haemolytic anaemia (AIHA) compared to control which indicated the catalase activity was attenuated in AlHA and lead to increased oxidative stress.

\section{Discussion}

In the present study we analysed the electrophoratogram of erythrocyte lysate for control and haemolytic samples. The overall protein content represented by intensity of bands revealed that in haemolytic anaemia most of the erythrocyte proteins were decreased in AIHA and NAIHA compared to control. One such protein identified in the $57 \mathrm{Kd}$ region was catalase, which was confirmed by mass spectrometry. Catalase is an important enzyme in the erythrocytes to convert $\mathrm{H}_{2} \mathrm{O}_{2}$ to $\mathrm{H}_{2} \mathrm{O}$ and $\mathrm{O}_{2}$. The bands visible in the region of $25 \mathrm{Kd}$ could be stomatin, aquaporin and peroxiredoxin and in the region of $15 \mathrm{Kd}$ was haemoglobin. All these bands showed decreased band intensity in the SDS-PAGE in haemolytic anaemia samples compared to control. In contrast, we also observed a band in the region near to 75 $\mathrm{Kd}$ which was observed only in Coombs test positive autoimmune haemolytic anaemia samples. This band was identified as albumin by mass spectrometry. The presence of albumin band in autoimmune haemolytic erythrocytes which was absent in control could not be rationalised to full extent in the scope of the current study. Nevertheless, the presence of premature erythrocytes or the reticulocytopenia or the presence of autoantibodies against erythrocyte antigens against immunoglobulin, IgG in AlHA could give an explanation for the presence of albumin in erythrocyte lysate. The possibility of plasma interference of albumin could be another possibility. But we did not find the presence of albumin in control or NAIHA samples which confirmed the specific presence of albumin in AIHA erythrocyte lysates. Albumin and globulin are the predominant plasma proteins which are capable of maintaining the morphological as well as physiological characteristics of erythrocytes. Albumin maintains the biconcave shape of the normal erythrocyte and decreases both osmotic and mechanical fragilities of erythrocytes (12). It was reported in a study that albumin had a synergistic effect with $y-$ globulin in decreasing erythrocyte sedimentation rate. The effect of albumin on the IgG induced and fibrinogen induced aggregation of human erythrocytes has shown that when albumin concentration in the medium was increased, the IgG induced erythrocyte aggregation was inhibited, while the fibrinogen induced erythrocyte aggregation was accelerated $(13,14)$.

\section{Conclusion}

In nut shell, the decreased presence of catalase protein and specific presence of albumin in autoimmune haemolytic anaemia erythrocyte lysate compared to control and non autoimmune anaemia are reported for the first time to the best of our knowledge. Further, we also observed there was an overall reduction in erythrocyte proteins in the molecular weight between $40 \mathrm{Kd}$ and $15 \mathrm{Kd}$ in response to haemolytic anaemia which could include aquaporin, stomatin, peroxiredoxin and haemoglobin.

\section{Acknowledgements:}

We are thankful to Central lab head Dr Susheela J Innah of the host Institute for providing the samples. We also would like to acknowledge $\mathrm{Dr}$ D.M. Vasudevan, Dr P.R. Varghese, Dr P.T. Annamala and all my colleagues for their valuable suggestions and academic support.

\section{References}

1. Freedman J. Autoimmune hemolysis: A journey through time. Transfusion Medicine and Hemotherapy. 2015;42(5):278-85.

2. Michel M. Warm autoimmune hemolytic anemia: advances in pathophysiology and treatment. La Presse Médicale. 2014;43(4): e97-104.

3. Packman $\mathrm{CH}$. Hemolytic anemia due to warm autoantibodies. Blood reviews. 2008;22(1):1731. 
4. Mayer B, Yürek S, Kiesewetter H, Salama A. Mixed-type autoimmune hemolytic anemia: differential diagnosis and a critical review of reported cases. Transfusion. 2008;48(10):2229-34.

5. Petz LD. Cold antibody autoimmune hemolytic anemias. Blood reviews. 2008;22(1):1-5.

6. Sallah S, Wan JY, Hanrahan LR. Future development of lymphoproliferative disorders in patients with autoimmune hemolytic anemia. Clinical cancer research. 2001;7(4):791-4.

7. Barcellini $W$, Fattizzo $B$, Zaninoni $A$, Radice $T$, Nichele I, Di Bona E, Lunghi M, Tassinari C, Alfinito F, Ferrari A, Leporace AP. Clinical heterogeneity and predictors of outcome in primary autoimmune hemolytic anemia: a GIMEMA study of 308 patients. Blood. 2014 Nov 6;124(19):2930-6.

8. Liesveld JL, Rowe JM, Lichtman MA. Variability of the erythropoietic response in autoimmune hemolytic anemia: analysis of 109 cases. Blood. 1987;69(3):820-6.

9. Conley $\mathrm{CL}$, Lippman SM, Ness P. Autoimmune hemolytic anemia with reticulocytopenia: $A$ medical emergency. JAMA. 1980;244(15): 1688-90.
10. Rosse WF. Quantitative immunology of immune hemolytic anemia: II. The relationship of cell-bound antibody to hemolysis and the effect of treatment. The Journal of clinical investigation. 1971;50(4):734-43.

11. Gehrs BC, Friedberg RC. Autoimmune hemolytic anemia. American journal of hematology. 2002;69(4):258-71.

12. Jay AW. Geometry of the human erythrocyte. I. Effect of albumin on cell geometry. Biophysical Journal. 1975;15(3):205-22.

13. Reinhart $W H$, Nagy C. Albumin affects erythrocyte aggregation and sedimentation. European journal of clinical investigation. 1995;25(7):523-8.

14. Maeda N, Shiga T. Opposite effect of albumin on the erythrocyte aggregation induced by immunoglobulin $G$ and fibrinogen. Biochimica et Biophysica Acta (BBA)-Biomembranes. 1986;855(1):127-35.

15. Arias M, Quijano JC, Haridas V, Gutterman JU, Lemeshko VV. Red blood cell permeabilization by hypotonic treatments, saponin, and anticancer avicins. Biochimica et Biophysica Acta (BBA)-Biomembranes. 2010;1798(6): 1189-96. 